\title{
GRADUAL TRAINING OF CASCADED SHAPE REGRESSION FOR FACIAL LANDMARK LOCALIZATION AND POSE ESTIMATION
}

\author{
Moh Edi Wibowo, Dian Tjondronegoro \\ Science and Engineering Faculty, Queensland University of Technology \\ moh.wibowo@student.qut.edu.au,dian@qut.edu.au
}

\begin{abstract}
Facial landmarks play an important role in face recognition. They serve different steps of the recognition such as pose estimation, face alignment, and local feature extraction. Recently, cascaded shape regression has been proposed to accurately locate facial landmarks. A large number of weak regressors are cascaded in a sequence to fit face shapes to the correct landmark locations. In this paper, we propose to improve the method by applying gradual training. With this training, the regressors are not directly aimed to the true locations. The sequence instead is divided into successive parts each of which is aimed to intermediate targets between the initial and the true locations. We also investigate the incorporation of pose information in the cascaded model. The aim is to find out whether the model can be directly used to estimate head pose. Experiments on the Annotated Facial Landmarks in the Wild database have shown that the proposed method is able to improve the localization and give accurate estimates of pose.
\end{abstract}

Index Terms - Facial landmark localization, pose estimation, cascaded shape regression

\section{INTRODUCTION}

Localization of facial landmarks is an important task in face recognition. It lies at the very core of face alignment, pose estimation, and feature extraction. To align faces, locations of eye centers are normally required. Similarity transform is then applied to rescale, rotate, and translate faces such that their eye centers reside at the same place. Head pose has been estimated using geometric methods which rely on facial landmarks. POSIT algorithm [1] for example, employs point correspondences between face images and a generic 3D face model to estimate head poses. Facial landmarks also constitute parts of the faces from where local features can be consistently extracted.

Facial landmarks have been localized using various methods. Among them, cascaded shape regression [2, 3] has shown remarkable performance. This method demonstrates high accuracy, runs in a considerably high speed, indicates
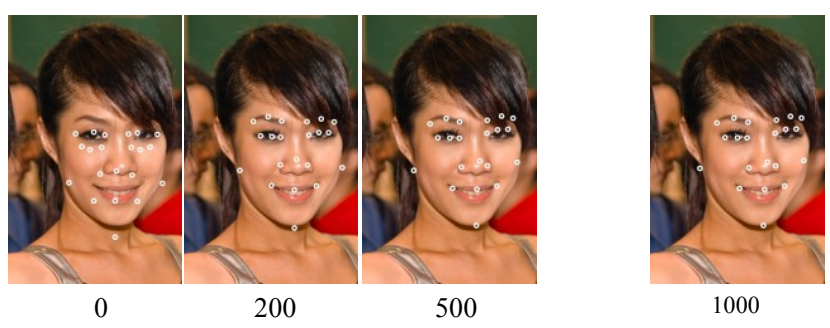

(a)

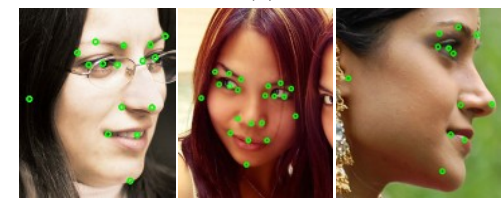

(b)

Fig. 1. (a) A face shape is fitted to a face image. From iteration 500 to 1000 the shape is not significantly changed. (b) Results of applying a single cascaded model to faces of different pose.

robustness to pose variation, and works well on real world data. The key of this method is probably the use of numerous weak regressors which together form a model with an accurate prediction capability. The model is used to successively fit a face shape to a face image. Each regressor is trained to predict shape increments required to update current shapes as close to the true locations as possible. Figure 1a shows the fitting of a face shape using the cascaded shape regression.

There is however, an interesting observation from the application of the cascaded shape regression. Despite the long sequence of the fitting, significant shape increments are only modeled by relatively few regressors at the beginning of the sequence (Figure 1a). The majority of the later regressors do not change the shape noticeably. It is thus imperative to find out whether spreading shape increments further across the sequence improves the performance or not. Another interesting note is the cascaded model seems to be able to learn data from different views. As shown in Figure $1 \mathrm{~b}$, face images of frontal, half-profile, and profile poses are well fitted by the same model. This opens an opportunity of using the model to predict head poses. 
In this paper, a gradual training of the cascaded shape regression is proposed. Normally, all regressors in the sequence are trained using true shapes of the faces as targets. In the gradual training though, intermediate targets are placed along the sequence before true shapes are placed as the final ones. Each regressor is then required to aim for intermediate targets which immediately come afterward. As can be seen from the experiments, this training method is able to improve performance of the model. Apart from the gradual training, we also investigate the possibility of using cascaded shape regression to directly estimate head pose. This is accomplished by appending shape vectors with elements corresponding to head rotations. It is then observed whether the shape fitting also leads to reasonably accurate predictions of such rotations.

The rest of the paper is organized as follows. Section 2 discusses related work. Section 3 describes the cascaded shape regression along with the proposed improvements. Section 4 presents experimental results. Finally, conclusions are drawn in Section 5.

\section{RELATED WORK}

In this section, some related methods of facial landmark localization will be discussed. The methods are roughly categorized into two approaches: localization by detection and iterative shape fitting.

Localization by detection searches for hypotheses of facial landmarks over various locations of input images and comes up with the final predictions of such landmarks. Such hypotheses have been found using template matching, neural networks, linear subspace or Gaussian modeling, boosted detectors [4] and generalized Hough transform for likelihood accumulation and voting $[5,6]$. Knowledge about face shapes is usually employed to guide the search or the selection of final predictions [5, 6, 7]. Limitation of this approach is it is computationally expensive especially when high accuracy is desired.

Another approach which is generally faster than landmark detection is the iterative shape fitting. Shapes in this context are vectors containing $x$ and $y$ coordinates of points corresponding to a number of predefined facial landmarks. To localize facial landmarks, an initial shape is placed to a face image and the shape is iteratively deformed. It is expected that the deformed shape will converge to the true locations of landmarks. Constrained local models (CLMs) refer to fitting methods which deforms shapes after estimating displacements of individual points independently [9]. These displacements are applied to the current shape subject to some shape constraints. Active shape models (ASMs) [8] are examples of these methods. ASMs obtain individual displacements by applying landmark detectors to the surrounding region of each point exhaustively. Point distribution models (PDMs) are used as shape constraints to avoid peculiar deformation. These later models comprise a number of orthogonal shape vectors learned from aligned samples using principal component analysis. These PDMs have also been employed in other similar methods such as in the regularized landmark mean-shift [9] and the regression voting method [10].

Apart from seeking individual point displacements, there are also fitting methods which estimate shape increments based on holistic face appearances. Linear regression has been employed in the classical active appearance models (AAMs) [11] to learn the mapping between regions occupied by the current shapes and shape increments. A gradient descent strategy i.e. the LucasKanade algorithm and non-linear regression have also been employed to improve these models $[12,13]$. Recently, an ensemble of weak regressors has been proposed to successively fit shapes to input images [2, 3]. Unlike AAMs which employ linear texture and shape models, the cascaded regression doesn't make use of any texture models and only encodes shape information implicitly within the mapping of the regressors. The method has demonstrated impressive robustness against real world variations and has become the state-of-the-art. This paper aims to improve the method by proposing a gradual training strategy and make use of the method in pose estimation.

\section{THE PROPOSED METHOD}

A cascaded regression model [2, 3] consists of $T$ weak regressors $\left(R^{1}, \ldots, R^{t}, \ldots, R^{T}\right)$. Each regressor takes a shape as input along with an image where the shape is fitted. It outputs a shape increment which is inferred from appearance features derived from the input. This ensemble of regressors determines the fitting of an initial shape $S^{0}$ to an image $I$. Given $S^{0}$ and $I$, the first regressor $R^{1}$ computes a shape increment $\delta S^{0}$ based on appearance features $\mathbf{x}^{0}$ extracted from image $I$. The shape $S^{0}$ is used as the coordinate reference of image $I$ when $\mathbf{x}^{0}$ is extracted. Once the increment is obtained, it is added to the current shape $S^{0}$ to produce $S^{1}$ which is then passed to the next regressor $R^{2}$. The same step is repeated until the last regressor $R^{T}$ is reached. This successive process can be expressed as

$$
\begin{aligned}
S^{t} & =S^{t-1} \oplus R^{t}\left(I, S^{t-1}\right) \\
& =S^{t-1} \oplus \delta S^{t-1}, \quad \text { for } t=1 \ldots T
\end{aligned}
$$

and the final shape $S^{T}$ can be written as

$$
S^{T}=\ldots\left(\left(S^{0} \oplus \delta S^{0}\right) \oplus \delta S^{1}\right) \oplus \ldots \oplus \delta S^{T-1}
$$

where the symbol $\oplus$ represents shape "addition". Note that image $I$ remains the same for all regressors. The feature vector $\mathbf{x}$ is also not recomputed at every $t$ instead it is recomputed once in a while as suggested in [2]. The regressors in between can just use the lastly recomputed 
(a)

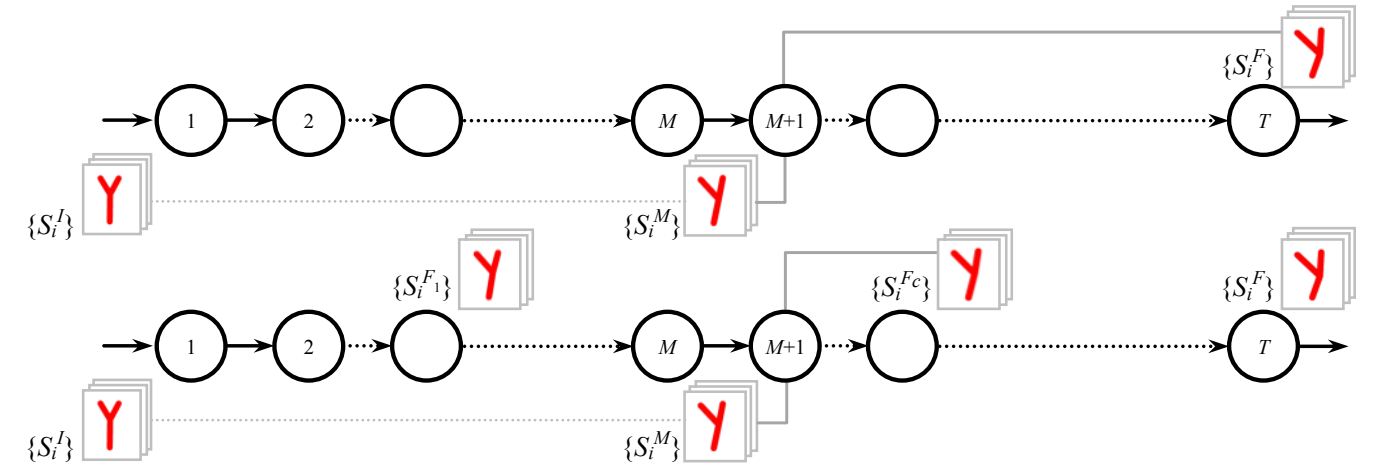

Fig. 2. (a) Regressor $R^{M+1}$ is trained using deformed shapes $\left\{S_{i}^{M}\right\}$ and true shapes $\left\{S_{i}^{F}\right\}$. (b) Regressor $R^{M+1}$ is trained using deformed shapes $\left\{S_{i}^{M}\right\}$ and intermediate target shapes $\left\{S_{i}^{F_{c}}\right\}$. Both training use the same image set $\left\{I_{i}\right\}$.

features. As the fitting proceeds, the shape $S^{t}$ is supposed to get closer to the true landmark locations.

To train the cascaded model, a set of face images $\left\{I_{i}\right\}$ and its corresponding sets of true shapes $\left\{S_{i}^{F}\right\}$ and initial shapes $\left\{S_{i}^{I}\right\}$ are required. True shapes are normally obtained from manual annotations while initial shapes can be generated heuristically. Suppose that $M$ regressors have already been trained successively and the set of initial shapes have been deformed into $\left\{S_{i}^{M}\right\}$ using (1). In the original cascaded model, regressor $R^{M+1}$ is trained using $\left\{I_{i}\right\},\left\{S_{i}^{F}\right\}$, and $\left\{S_{i}^{M}\right\}$ as shown in Figure 2a. In this paper, we propose to train this regressor using $\left\{I_{i}\right\},\left\{S_{i}^{F^{c}}\right\}$, and $\left\{S_{i}^{M}\right\}$ where $\left\{S_{i}^{F_{c}}\right\}$ is the $c$-th set of intermediate targets defined in between the initial and the true shapes:

$$
S_{i}^{F_{c}}=((C-c) / C) S_{i}^{I}+(c / C) S_{i}^{F} .
$$

$C$ is the number of sets of intermediate targets i.e. $c=1 \ldots$ $C$. Note that these sets are placed along the sequence of regressors and for regressor $R^{M+1}$ the set $\left\{S_{i}^{F_{c}}\right\}$ is the one immediately comes afterward. This training strategy is shown in Figure 2b. The aim of such a gradual training is to spread shape increments more equally across the sequence of regressors.

There are several aspects that need to be specified for the cascaded model e.g. some operations on shapes, the appearance features, and the weak regressors.

\subsection{Shape and shape operations}

A shape $S$ can be written as

$$
S=\left(x_{1}, y_{1}, x_{2}, y_{2}, \ldots, x_{N}, y_{N}\right)
$$

where $N$ is the number of predefined landmarks. These vectors contain two types of variations: (i) global positions, scales, and rotations, and (ii) local shape variations including those due to change of head pose.
Two binary operations are defined for the shapes. Given two shapes $S$ and $S$, the "subtract" operation $S^{\prime}-S$ calculates a shape increment $\delta S$ required to deform $S$ to $S^{\prime}$. Given a shape $S$ and a shape increment $\delta S$, the "add" operation $S \oplus \delta S$ computes the resulting shape $S^{\prime}$ when the increment $\delta S$ is applied to $S$. These two operations are needed later by the cascaded model. One thing to remember is these operations should disregard any effects caused by the global shape transform. One way to achieve this is transforming all rectangular face regions (e.g. ones returned by face detectors) into same sizes and positions. In this case, the "subtract" and "add" operations of the shapes can be defined as normal vector addition and subtraction respectively. Another method which is used in this paper is employing shape alignment using a single reference shape. If $\mathrm{T}_{S}$ is a transform aligning $S$ to a reference shape $\mathrm{R}, \delta S=S^{\prime}$ $-S$ can be defined as $\mathrm{T}_{S}\left(S^{\prime}\right)-\mathrm{T}_{S}(S)$ and $S^{\prime}=S \oplus \delta S$ can be defined as $\mathrm{T}_{S}^{-1}\left(\mathrm{~T}_{S}(S)+\delta S\right)$. Least square method is employed to compute similarity transform between shapes.

\subsection{Appearance features}

Intensity difference of two image pixels is the commonly adopted feature for cascaded regression. It is cheap to compute but quite powerful given the training data are sufficient enough. The feature vector $\mathbf{x}$ then consists of a number of such features. A set of offset pairs $\left(j_{i}{ }^{1}, x_{i}{ }^{1}, y_{i}{ }^{1}, j_{i}{ }^{2}\right.$, $\left.x_{i}^{2}, y_{i}^{2}\right)_{i=1}^{F}$ is randomly generated at some $t$ s of the cascaded model. Remember that appearance features are not extracted at every $t$. Given such a set of offset pairs, a shape $S^{t}$, and an image $I$, the features can be extracted by first "adding" offset pairs to $S^{t}$ to obtain image coordinates $\left(x x_{i}{ }^{1}, y y_{i}{ }^{1}, x x_{i}{ }^{2}, y y_{i}{ }^{2}\right)_{i=}{ }^{F}$ ${ }_{1}$ and then computing intensity differences

$$
\mathbf{x}_{i}^{t}=I\left(x x_{i}^{1}, y y_{i}^{1}\right)-I\left(x x_{i}^{2}, y y_{i}^{2}\right), \quad i=1 \ldots F
$$

To "add" offsets to the shape, we treat the offsets as if they are shape increments. The index $j$ indicates to which point of 
the face shape the $x$ and $y$ offsets have to be added. Note that this feature extraction procedure is invariant against global position, scale, and rotation.

\subsection{Random fern}

Random fern is the weak regressor employed in the cascaded model. A fern is actually a complete binary decision tree whose splitting nodes of the same level employ the same test function. A fern of depth $D$ will thus evaluate $D$ features from the feature vector $\mathbf{x}$. At the bottom of this fern, there will be $2^{D}$ bins to each of which a particular output is associated. Given a set of training cases $\left\{\left(\mathbf{x}_{i}, \delta S_{i}\right)\right\}$, each case consists of a feature vector $\mathbf{x}$ and a target value $\delta S$, the fern can be trained using a simple algorithm [3]:

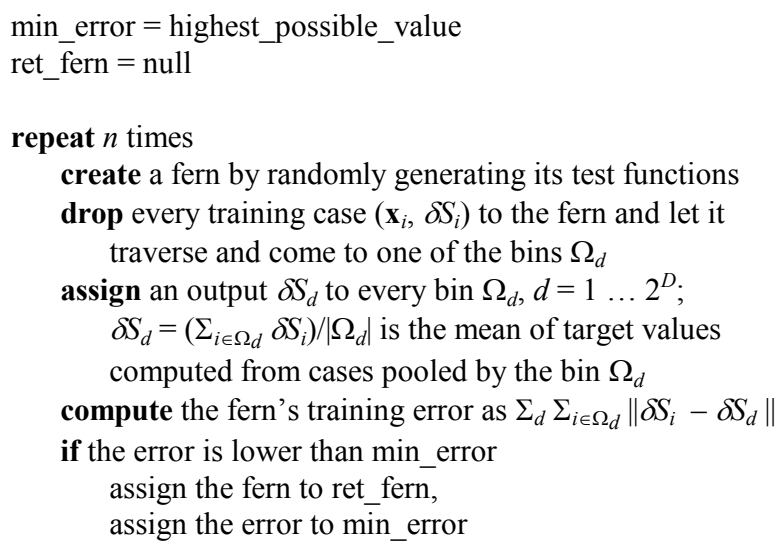

return ret_fern

The above algorithm basically generates a number of ferns randomly and then chooses the one which has the minimum training error. Note that the set of training cases $\left\{\left(\mathbf{x}_{i}, \delta S_{i}\right)\right\}$ for a fern $R^{t}$ is computed from the true shapes $\left\{S_{i}^{F}\right\}$, deformed shapes $\left\{S_{i}^{M}\right\}$, and images $\left\{I_{i}\right\} . S_{i}^{F}$ and $S_{i}^{M}$ are used to obtain $\delta S_{i}$ using the "subtract" operation while $I_{i}, S_{i}^{M}$, and the randomly generated offset pairs are used to obtain $\mathbf{x}_{i}$. For most ferns however $\mathbf{x}_{i}$ is not re-computed but simply obtained from the previous step. The random offset pairs need to be saved and they will be required in the testing stage. Shrinkage is also often applied in the computation of $\delta S_{d}[2]$.

\subsection{Fitting of multiple instances}

To localize facial landmarks in a test image, multiple instances of face shape are fitted using the cascaded model. In this paper, 8 initial shapes are first fitted to the image. Following this, a clustering procedure is applied to the fitted shapes. If there is no cluster with 4 or more members, more initial shapes are fitted to the image until such a cluster is obtained or until 16 shapes have been fitted. Members of the

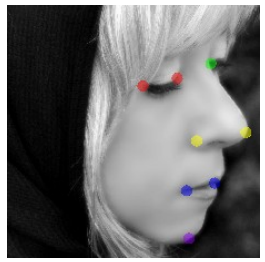

(a)

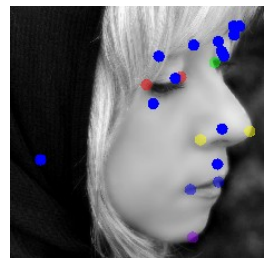

(b)

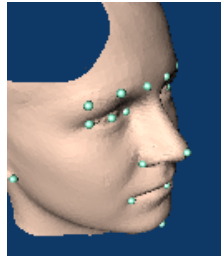

(c)
Fig. 3. POSIT algorithm is applied to a face with missing landmarks (a) to obtain estimates of their locations (b). The aligned 3D face model is also shown in (c).

biggest cluster are then averaged to get the final result of the localization.

Incremental agglomeration is employed for the clustering. Each time a shape $S$ gets fitted, the shape will be checked whether it is "connected" to some of the existing clusters. $S$ is "connected" to a cluster $C$ if $C$ has a member $Z$ whose at least $\lambda \times 100 \%$ of its points are within a distance $\sigma$ from the corresponding points of $S$. In other words, $S$ is connected to $C$ if there is a $Z \in C$ and

$$
\frac{\sum_{i=1 \ldots N} h\left(\left\|S^{i}-Z^{i}\right\|, \sigma\right)}{N} \geq \lambda
$$

where

$$
h\left(\left\|S^{i}-Z^{i}\right\|, \sigma\right)=\left\{\begin{array}{l}
1, \text { if }\left\|S^{i}-Z^{i}\right\| \leq \sigma \\
0, \text { otherwise }
\end{array}\right.
$$

If some clusters are connected to $S$, these clusters and $S$ are merged into one. Otherwise, $S$ will form a new cluster.

The clustering procedure is not applied directly to the fitted shapes. It is instead applied to normalized shapes obtained from standardizing the position and scale of the fitted shapes based on the bounding box of the face. This way, the clustering procedure is not required to handle scale variations and its parameters do not need to be adaptive. To normalize the shapes, a rescaling factor $r$ and shifts $(\Delta x, \Delta y)$ which change the bounding box to a $320 \times 320$ box positioned at $(0,0)$ are computed. The normalization then moves each point $(x, y)$ of a fitted shape $S$ to $((x-\Delta x) \times r,(y$ $-\Delta y) \times r)$.

\subsection{Pose estimation}

To estimate head pose using the cascaded model, we train the model using data containing pose information. Shape vectors are simply appended with elements containing rotations of the head. The "add" and "subtract" operations are defined on these elements as the normal addition and 
subtraction respectively. To train the regressors with the new data, we use the exactly same procedure as before. We do not change the computation of ferns' errors meaning that the regressors are not directly attuned to head rotations during the training. In our experiments, we are particularly interested to estimate "left-right" (yaw) rotations.

\section{EXPERIMENTS}

\subsection{Training}

The Annotated Facial Landmarks in the Wild (AFLW) database [14] is employed in the experiments. We use 2877 face images from the database as the training set. These images are selected such that they cover large variations of pose and rotation. There are up to 21 landmarks annotated within each image. Annotations of head pose and face's bounding box are also available. Due to pose and occlusion, some landmarks may not be visible and the corresponding annotations are not available. To solve this issue, we employ POSIT algorithm to estimate missing locations using the known ones. This algorithm utilizes a generic 3D face model whose viewing parameters are solved appropriately based on $3 \mathrm{D}$ to $2 \mathrm{D}$ point correspondences. Figure 1 shows images from the AFLW database some of which are imposed with the 21 points. Figure 3 shows a face with missing landmarks, the predicted locations of landmarks, and the aligned 3D face model after the execution of POSIT algorithm.

At this point, the other things we need to prepare are the initial shapes. We simply use the average face shape centered to the bounding box of the face as the initial shape of each image. To achieve a better generalization, each initial shape is augmented 11 times by perturbing it with up to $15^{\circ}$ of rotation, $10 \%$ of shift, and $8 \%$ of scaling. The cascaded model is then trained by setting the number of regressors to 1000 , the depth of the ferns to 8 , the number of appearance features to 400 , and these features are only recalculated every 30 steps. We use 5 sets of intermediate targets (including the set of true landmark locations) and place a new set at the sequence after 200 regressors are passed. Regressors in the cascaded models are trained successively. Every time a set of intermediate targets is passed, some of the deformed (initial) shapes are perturbed.

\subsection{Generating initial shapes}

As previously mentioned, multiple initial shapes are fitted to a test image to localize facial landmarks. There can be different ways to generate such initial shapes. For example, an average face shape can be centered to the face's bounding box and the centered shape is perturbed several times. In our work however, initial shapes are derived by employing misalignments of bounding boxes detected by face detectors and bounding boxes used to train the cascaded model (bounding boxes specified by the manually annotations).
Note that we use Viola-Jones face detectors provided by OpenCV library in our experiments. The misalignments are learned by computing three correction values $(d x, d y, s c)$ where

$(d x, d y)=$

left-top corner of man. annotation - left-top corner of the detection width of the detection

$s c=\frac{\text { width of man. annotation }}{\text { width of the detection }}$.

Having collected such correction values from training data, it turns out that the values can be modeled using a single mode multivariate Gaussian distribution. Given a test image and its automatically detected face's bounding box, a number of "corrected" bounding boxes can be generated by applying "random corrections" drawn from the distribution. Average face shapes can then be centered to the "corrected" bounding boxes to obtain initial shapes for the image.

\subsection{Evaluation results}

To evaluate the trained models, 1237 images of the AFLW database are used as test data. These images are selected such that the faces can be detected using Viola-Jones face detectors. To give quantitative results, the number of images on which the fitting runs successfully is counted. A fitting process is said to be successful if all points of the final shape are within a certain distance from their true locations. For each facial landmark, point-to-point error can be defined as $e=\|\mathbf{p}-\tilde{\mathbf{p}}\| \times s$ where $\mathbf{p}$ refers to the localized position, $\tilde{\mathbf{p}}$ represents the ground truth position, and $s$ is a normalizing factor. Traditionally, $s$ is the inverse of the eye-to-eye (interoccular) distance. In this work however $s$ is chosen as the inverse of the true distance between inner tip of the eyebrow and center of the mouth. Note that this later normalizing factor is also suitable for non frontal faces whose eyes might not be both visible. Successful fitting is concluded if all point-to-point errors of the final shape are less than a threshold $\tau$. The value of threshold $\tau$ is varied and the percentages of successful fitting are plotted.

Figure $4 \mathrm{a}$ and $4 \mathrm{~b}$ show localization results of the standard and the gradually trained cascaded models when $\lambda$ is set to $\{0.6,0.65, \ldots, 0.95\}$ and $\sigma$ is set to $\{10,12, \ldots$, $20\}$ pixels. Both models achieve optimum performance when $\lambda$ is set to 0.6 and $\sigma$ is set to 16 pixels. It is observed that the gradually trained model gives better optimum performance than the standard cascaded model. This is more clearly shown in Figure 4c. When the threshold $\tau$ is set to $0.2,0.25$, and 0.3 , the gradually trained model achieves $50.6 \%, 76.8 \%$, and $86.1 \%$ of successful fitting respectively. The standard cascaded model on the other hand achieves $39.0 \%, 65.3 \%$, and $77.8 \%$ of successful fitting. 


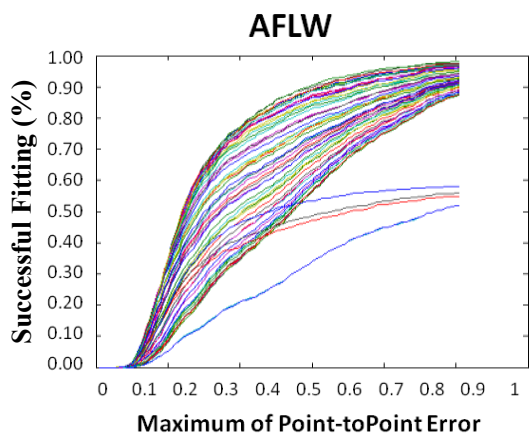

(a)

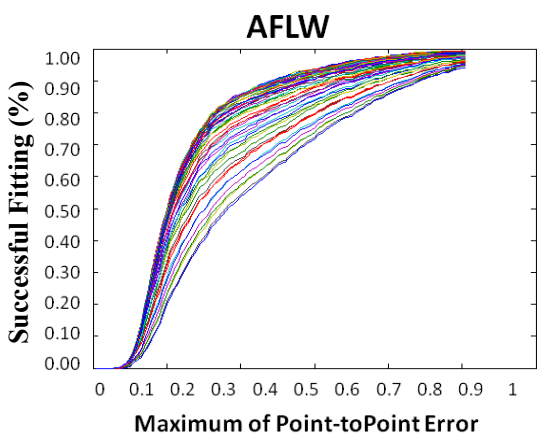

(b)

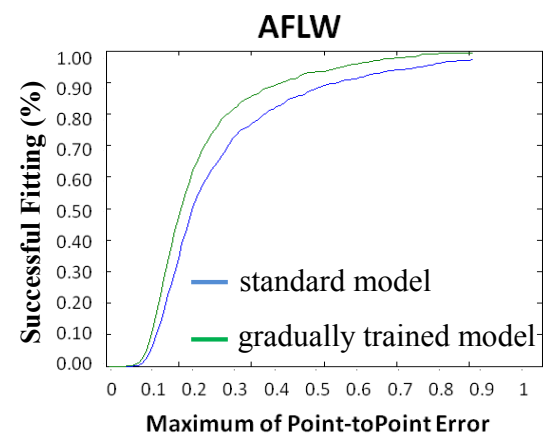

(c)

Fig. 4. Percentages of successful fitting, plotted against different values of maximum of point-to-point errors $\tau$, shown for: (a) the standard cascaded model and (b) the gradually trained cascaded model. Each line in the graph corresponds to a particular pair of $\lambda$ and $\sigma$ values. When $\lambda$ and $\sigma$ are set to 0.6 and 16 respectively, the gradually trained model outperforms the standard model as shown in (c).

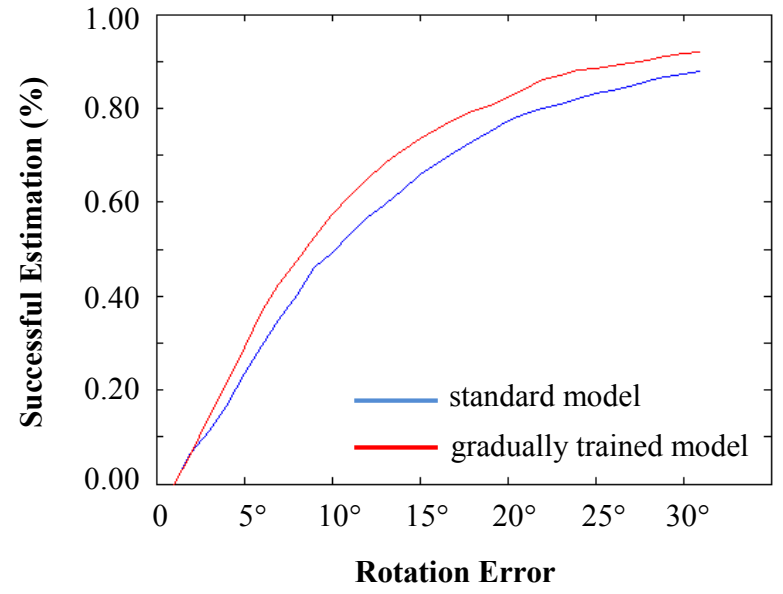

Fig. 5. Percentages of successful pose estimation, plotted against different values of rotation error.

Figure 5 shows the results when both cascaded models are employed to estimate head pose. It is shown that $68.4 \%$ of the test data can be estimated with less than $15^{\circ}$ of rotation error using the standard model. With the gradually trained model, $75.5 \%$ of the test data can be estimated within the same error threshold. The means of estimation errors of the standard and gradually trained models are $14.78^{\circ}$ and $11.87^{\circ}$ respectively. Note that these means are computed using all test data.

\section{CONCLUSION}

We propose a gradual training of the cascaded shape regression for facial landmark localization. Instead of training all regressors using the true shapes, we set a number of intermediate targets along the cascade. Each regressor is then required to learn from the next immediate targets. We also extend the cascaded model to perform pose estimation. We append head rotations to shape vectors such that the model may infer head poses in addition to face shapes. Experiments on the AFLW database have shown that landmark localization can be improved by applying the gradual training. The experiments have also shown that the cascaded model can be used to estimate continuous head poses.

\section{REFERENCES}

[1] D. F. Dementhon and L. S. Davis, "Model-based object pose in 25 lines of code," International Journal of Computer Vision, vol. 15, pp. 123-141, 1995.

[2] X. Cao, Y. Wei, F. Wen, and J. Sun, "Face alignment by explicit shape regression," in IEEE Conference on Computer Vision and Pattern Recognition (CVPR) 2012, 2012, pp. 2887-2894.

[3] P. Dollar, P. Welinder, and P. Perona, "Cascaded pose regression," in IEEE Conference on Computer Vision 
and Pattern Recognition (CVPR) 2010, 2010, pp. 10781085.

[4] D. Vukadinovic and M. Pantic, "Fully automatic facial feature point detection using Gabor feature based boosted classifiers," in IEEE International Conference on Systems, Man and Cybernetics 2005, 2005, pp. 1692-1698.

[5] M. Valstar, B. Martinez, X. Binefa, and M. Pantic, "Facial point detection using boosted regression and graph models," in IEEE Conference on Computer Vision and Pattern Recognition (CVPR) 2010, 2010, pp. 2729-2736.

[6] M. Dantone, J. Gall, G. Fanelli, and L. Van Gool, "Real-time facial feature detection using conditional regression forests," in IEEE Conference on Computer Vision and Pattern Recognition (CVPR) 2012, 2012, pp. 2578-2585.

[7] P. N. Belhumeur, D. Jacobs, D. J. Kriegman, and N. Kumar, "Localizing parts of faces using a consensus of exemplars," in IEEE Conference on Computer Vision and Pattern Recognition (CVPR) 2011, 2011, pp. 545552.

[8] T. F. Cootes, C. J. Taylor, D. H. Cooper, and J. Graham, "Active shape models - their training and application," Computer Vision and Image Understanding, vol. 61, pp. 38-59, 1995.
[9] J. M. Saragih, S. Lucey, and J. F. Cohn, "Deformable model fitting by regularized landmark mean-shift," International Journal of Computer Vision, vol. 91, pp. 200-215, 2011.

[10] T. Cootes, M. Ionita, C. Lindner, and P. Sauer, "Robust and accurate shape model fitting using random forest regression voting," in European Conference on Computer Vision (ECCV) 2012, pp. 278-291, 2012.

[11] T. F. Cootes, G. J. Edwards, and C. J. Taylor, "Active appearance models," IEEE Transactions on Pattern Analysis and Machine Intelligence, vol. 23, pp. 681$685,2001$.

[12]I. Matthews and S. Baker, "Active appearance models revisited," International Journal of Computer Vision, vol. 60, pp. 135-164, 2004.

[13]P. Sauer, T. Cootes, and C. Taylor, "Accurate regression procedures for active appearance models," in Proceedings of the British Machine Vision Conference, 2011, pp. 30.1-30.11.

[14] M. Kostinger, P. Wohlhart, P. M. Roth, and H. Bischof, "Annotated facial landmarks in the wild: A large-scale, real-world database for facial landmark localization," in IEEE International Conference on Computer Vision Workshops (ICCV Workshops) 2011, 2011, pp. 21442151. 